\title{
LIBER8 Design and Methods: An Integrative Intervention for Loss of Control Eating among African American and White Adolescent Girls
}

\author{
Suzanne E. Mazzeo ${ }^{\mathrm{a}, \mathrm{b}}$, Nichole R. Kellya ${ }^{\mathrm{a}}$, Marilyn Stern ${ }^{\mathrm{a}, \mathrm{b}}$, Allison A. Palmberg ${ }^{\mathrm{a}}$, Faye Z. \\ Belgrave $^{\mathrm{a}}$, Marian Tanofsky-Kraff ${ }^{\mathrm{c}}$, Yael Latzer, and Cynthia M. Bulik \\ aDepartment of Psychology, Virginia Commonwealth University, Richmond, VA \\ ${ }^{b}$ Department of Pediatrics, Virginia Commonwealth University, Richmond, VA \\ 'Department of Medical and Clinical Psychology, Uniformed Services University of the Health \\ Sciences, Bethesda, MD \\ dDepartment of Social Welfare and Health Studies, University of Haifa, Haifa, Israel \\ eDepartment of Psychiatry, University of North Carolina at Chapel Hill, Chapel Hill, NC
}

\begin{abstract}
Loss of control (LOC) eating affects a significant number of adolescents of all racial and ethnic backgrounds and is associated with numerous psychosocial problems, including depression, anxiety, low self-esteem, body dissatisfaction, and weight concerns. However, empirically validated, culturally sensitive treatments for adolescents with these disordered eating behaviors are not available. This pilot project involved designing a developmentally and culturally appropriate treatment for LOC eating for adolescent girls. We intend to conduct multiple focus groups with adolescent girls who engage in LOC eating, and their primary caregivers. Data from these groups will inform the subsequent creation of a manualized treatment protocol. We will then evaluate the efficacy of this intervention (LIBER8 - Linking Individuals Being Emotionally Real) to reduce LOC eating. This intervention will integrate components of dialectical behavior therapy, such as mindfulness and distress tolerance skills training, and cognitive-behavioral therapy. We will also integrate text-messaging, a key adolescent communication strategy, as a means of self-monitoring. Participants meeting study criteria will be offered participation in this 12-week randomized controlled trial comparing LIBER8 to a weight management control condition (2BFit). We hypothesize that this intervention will serve to reduce LOC eating, as well as improve psychosocial functioning as evidenced by decreased depression, anxiety, eating disorder cognitions, emotional eating, impulsivity, and improved quality of life. The feasibility and
\end{abstract}

(C) 2012 Elsevier Inc. All rights reserved.

Designated Author for Correspondence: Suzanne Mazzeo, Ph.D. Virginia Commonwealth University 806 West Franklin St. P.O. Box 842018 Richmond, Virginia 23284-2018 804.827.1708 semazzeo@vcu.edu.

ClinicalTrials.gov \#: NCT01395355

Publisher's Disclaimer: This is a PDF file of an unedited manuscript that has been accepted for publication. As a service to our customers we are providing this early version of the manuscript. The manuscript will undergo copyediting, typesetting, and review of the resulting proof before it is published in its final citable form. Please note that during the production process errors may be discovered which could affect the content, and all legal disclaimers that apply to the journal pertain. 
acceptability of this intervention will be extensively evaluated with the explicit intent of informing a subsequent larger randomized controlled trial.

\section{Keywords}

binge eating disorder; loss of control; adolescents; African American; treatment; randomized clinical trial

\section{Introduction}

\subsection{Significance}

Binge eating disorder (BED) is the most common eating disorder among adults, occurring in $3.5 \%$ of women and $2.0 \%$ of men [1]. BED is typically chronic and is associated with significant impairment in daily functioning and obesity [1,2]. Many adults with BED report that their symptoms began in childhood [3]. Thus, the development of effective interventions targeting binge eating in youth could reduce the significant impact of BED on quality of life over the lifespan.

\subsection{Binge Eating among Adolescents}

Nearly a third of adolescents seeking obesity treatment report binge eating behavior [4-7]. Binge eating in youth is associated with numerous psychosocial problems, including depression, anxiety, and body dissatisfaction $[4,5,7,8]$. Further, the association between binge eating and distress has been found in both treatment-seeking $[4,9]$ and non-treatment seeking $[10,11]$ adolescents, and in diverse ethnic and racial groups [4, 5]. Moreover, although binge eating is associated with obesity in adolescence [7, 12], studies have suggested that binge eating severity is a stronger influence on psychosocial functioning than the severity or duration of obesity $[4,5,7,10]$. Thus, adolescents who engage in binge eating appear to be particularly vulnerable to psychological distress which does not appear to be attributable solely to their weight status. Binge eating is also predictive of excess weight gain [13-15] and psychological distress over time [16].

\subsection{Binge Eating is Difficult to Define and Measure in Youth}

Despite its public health significance, binge eating is difficult to assess. Although several measures exist to evaluate binge eating in youth [17-19], concerns about their validity remain [20-22]. Traditional adult assessments of binge eating require that a binge episode be identified only if the amount of food consumed is deemed "unambiguously large" [23]. Given the varying energy needs of physically developing children, it is often difficult to make this determination. Moreover, as noted by Goossens et al. [24], children might not have access to large amounts of food. Thus, although their eating might be uncontrolled, an unambiguously large amount of food might not be consumed because it is simply unavailable.

Therefore, researchers working with children have generally focused on the experience of LOC during eating, regardless of whether the reported amount of food consumed is unambiguously large $[22,24]$. Thus, the term LOC eating, as opposed to binge eating, is 
often adopted when working with children and adolescents to be inclusive of all episodes involving a sense of losing control. LOC eating, regardless of the amount of food consumed, is associated with adverse psychological and physical correlates and outcomes [4, 24-26]. Finally, no differences in LOC behavior have been identified across ethnic or racial groups, suggesting that these problematic eating styles affect African American, White, and Hispanic adolescents equally [5, 11]. In summary, LOC eating is prevalent among adolescents and is an important risk factor for psychopathology. However, there are few empirically-based treatments for LOC eating for this age group.

\section{Objectives of LIBER8}

This paper aims to describe the rationale for and design of an intervention [LIBER8Linking Individuals Being Emotionally Real] for adolescent girls targeting LOC eating that integrates components of dialectical behavior therapy (DBT), such as mindfulness and distress tolerance skills training [27] and cognitive-behavioral therapy (CBT) [28, 29]. Adolescents will self-monitor by text messaging-a key component of youth communication-as an integrated intervention delivery tool to reduce LOC frequency. The resulting intervention will be culturally targeted to enhance its applicability to our primarily African-American sample.

The proposed project has three Specific Aims: (1) To develop and standardize a manualized intervention (LIBER8) for adolescents with LOC eating behavior to facilitate therapist training and treatment dissemination; (2) To pilot LIBER8 in a small, randomized controlled trial (RCT) to determine feasibility and acceptability; and (3) To evaluate whether LIBER8 is superior to a healthy eating control intervention (2BFit) for improving targeted outcomes. These exploratory analyses will be conducted to inform the design of a subsequent largescale RCT.

\section{Study Design}

\subsection{Overview}

The proposed project is comprised of three stages. In Stage 1, focus groups will be conducted with adolescents who endorse LOC eating behaviors; their parents/caregivers will also participate in parallel (yet separate) focus groups. Data derived from these groups will be used to develop a pilot version of the LIBER8 intervention. During this stage, the text messaging program will also be configured. Based on our previous research in the Richmond community, and the aim of this project to enhance understanding of LOC eating in African American girls, we anticipate that the majority of participants in Stage 1 (and Stages 2 and 3), will be African American. However, race/ethnicity will not be an inclusion criterion in any stage of this study.

In $\underline{\text { Stage 2, }}$, we will use the data obtained in $\underline{\text { Stage } 1}$ to develop a manual for the intervention. We will solicit feedback on the acceptability and feasibility of the manualized intervention from four to six adolescents who endorse LOC eating. Adolescent reviewers will be asked to rate the manual on the following dimensions: (1) organization and presentation, (2) content (e.g. appropriateness, order of topic presentation), (3) cultural sensitivity, (4) therapeutic 
level of the presentation, (5) sensitivity to issues faced by adolescents with LOC eating behaviors, (6) user-friendliness, (7) quality and believability of clinical vignettes, (8) usefulness and quality of worksheets and homework assignments, and (9) visibility of contact information and emergency procedures to be used during the clinical trial. In addition, we will pilot our assessment instruments and procedures [including text messaging) with the adolescents. Finally, we will ask clinicians with experience treating adolescents with LOC eating to provide their feedback on the acceptability and feasibility of the manual and procedures. All feedback will be compiled and the manual development team will meet to determine final changes to the LIBER8 manual. This work will yield a finalized version of the intervention manual to be implemented in Stage 3. This combination of information from adolescents, clinicians, and experts in developing eating disorder interventions combines three powerful vantage points to develop an optimal intervention $[30,31]$.

In $\underline{\text { Stage } 3}$, we will conduct a pilot RCT of the LIBER8 intervention $(n=25)$ versus 2 BFit $(n=25)$ for adolescents with current LOC eating behaviors. Assessments will be conducted at baseline, mid-treatment, post-treatment, and three month follow-up. The primary outcome is LOC eating behavior. Secondary outcomes include eating disorder cognitions, emotional eating, emotion regulation, depression, anxiety, impulsivity, and quality of life. The feasibility and acceptability of the intervention, as well as text messaging as a selfmonitoring tool, will also be assessed. Three audiotapes from each group will be rated for fidelity to the treatment manual by research staff. Findings from this small RCT will yield effect sizes to inform a subsequent large-scale RCT.

\subsection{Intervention Model Rationale}

Cognitive Behavioral Treatment for Binge Eating-There are several reasons why empirical evaluation of the efficacy of CBT for LOC eating in adolescents is warranted. First, CBT is considered the "gold standard" of treatment for eating disorders in adults, particularly bulimia nervosa [32,33]. Moreover, in a review of the effectiveness of BED treatment with adults, Brownley et al. [34] reported that CBT was moderately effective in reducing binge frequency and related eating attitudes and behaviors, such as disinhibition and restraint. Secondly, treatments based on this theoretical approach have effectively reduced symptomatology among adolescents with other psychiatric diagnoses, such as depression [35]. Thirdly, CBT directly targets specific maladaptive behaviors, such as restrictive dieting. This is important, as empirical studies have found that restrictive dieting precedes binge and LOC eating for approximately $35 \%$ of youth who engage in these disordered eating behaviors [25]. Thus, a CBT-based approach should be effective in reducing the problems of LOC eating, as well as other specific maladaptive behaviors, among adolescents.

Dialectical Behavior Therapy for Binge Eating-DBT is another promising approach for the treatment of LOC eating behavior [27]. This therapeutic approach emphasizes emotion regulation and distress tolerance skills training. It has been proposed that these skills would be useful for other clinical problems, including eating disorders, in which maladaptive coping mechanisms are used to manage negative affect [36, 37]. Moreover, this 
treatment approach is consistent with the affect regulation model of binge eating behavior described by Wiser and colleagues [38] which attributes binge eating to deficits in emotion regulation skills.

In addition to its relevance to LOC eating behavior, training in distress tolerance and emotion regulation skills is also particularly appropriate for adolescents. This developmental period is a pivotal time for the expansion of individuals' coping repertoire, as emerging cognitive skills can facilitate adolescents' ability to use a wider range of coping strategies [39]. Thus, the proposed intervention will include training in distress tolerance, mindfulness, and emotion regulation.

\subsection{Engaging Adolescents in Treatment with Text-Messaging}

The problem of treatment nonadherence among adolescents with chronic health conditions is well-known, and is associated with significant morbidity [40, 41]. Many strategies for enhancing adherence among adolescents have been recommended. The incorporation of technological tools that are frequently used by adolescents is one approach that holds particular promise [42]. Text-messaging may incorporate technology and structure into an intervention. To date, research has not examined the use of text-messaging in the treatment of LOC eating in adolescents. Adding this element to interventions targeting adolescents seems promising, as texting is a preferred form of communication in this age group.

Previous studies have applied texting to health care interventions [43-47]. Studies suggest that youth prefer texting over other forms of communication because they feel they have more control over conversations and can take time before responding [48]. Other studies have found that participants prefer to share personal and sensitive information virtually as they are less concerned with the moderator's opinion of them [49,50]. Texting can also serve as a convenient method of self-monitoring. Behavioral theory defines self-monitoring as central to behavior change because it enhances learning about patterns and promotes adherence to goals. Despite the efficacy of self-monitoring, adherence is often poor for both children and adults [51-54].

In the current study, we will use texting to facilitate the self-monitoring component of LIBER8. Given adolescents' comfort with technology, the frequency of cell phone use, the promising results of text-messaging pilot studies [44, 55], the behavioral principles supported by immediate feedback [56, 57], and problems with adherence to traditional monitoring, we are studying text-messaging as a promising and innovative approach to decrease LOC eating behaviors. We will configure the texting program to accommodate feedback for self-monitoring of symptoms associated with LOC eating including: (1) number of LOC episodes, (2) number of overeating episodes; (3) urges to overeat, and (4) intensity of overeating urges. Thus, this self-monitoring component will enable the research team both to communicate with participants and will also serve the purpose of capturing study data.

\subsection{Enhancing the Cultural Sensitivity of Interventions for African American Adolescents}

LIBER8 will address a critical gap in the literature on the treatment of LOC eating by focusing on the needs of African American adolescents, a group at high risk for these eating 
disorder behaviors [58-61]. Studies of pediatric samples suggest that binge eating is a significant problem among African American adolescents [4, 5]. Further, African American youth who engage in LOC eating report clinical impairment comparable to that of their White peers on measures of quality of life, depression, anxiety, and self-esteem, suggesting that ethnicity does not provide any buffering effect against the negative effects of this eating disorder $[4,5,62]$.

Further, a recent report by the American Psychological Association [63], notes that challenges more commonly faced by urban African American youth, such as poverty and neighborhood violence, are associated with emotion regulation difficulties. Cultural differences in emotional expression norms might have important implications for interventions addressing affect regulation [63]. Further, these authors suggest that the Africenteric strengths of expressiveness, spirituality, and communalism should be considered in the development of interventions targeting African American youth. Studies in other areas of adolescent mental health including substance abuse [64, 65], have found that culturally relevant interventions yield positive outcomes with African American adolescent girls. However, no studies have investigated the efficacy of eating disorder treatment that was designed to be culturally sensitive, in either African American adults or children.

Enhancing the cultural sensitivity of interventions is also important because food selection, preparation, and the eating process itself (including how and with whom food is eaten) are significantly influenced by cultural factors [66, 67]. Additionally, food availability is often dependent on context and many African American youth live in under-resourced communities where food choices are poor. Thus, interventions need to be sensitive to these concerns, and highlight the health-promoting aspects of Africentric values and customs related to food selection and eating behavior. Moreover, research on interventions promoting healthy eating in adult women $[68,69]$ found that African Americans preferred a group (versus individual) approach. This is consistent with the collectivist orientation of many African Americans [68].

The cultural sensitivity of LIBER8 will be enhanced via its focus on acknowledging and incorporating racial and ethnic differences in body image ideals, building peer and family support for behavior change (versus using an individual approach), and incorporating aspects of spirituality [70]. The group format of the intervention is also consistent with the communal, collectivist orientation of the African American community, which emphasizes interpersonal relationships, and the value placed on expressive communication [67, 68]. Further, the examples provided in the LIBER8 intervention will address family relationships, and the range of family structures represented in African American culture. It will also acknowledge differences in parenting styles across racial groups, and how these differences might affect adolescents' attempts to establish autonomy, particularly in relation to eating habits and emotion regulation.

Nonetheless, it should be noted that members of other ethnic/racial groups will be allowed to enroll in LIBER8. This could mitigate potential concerns about the external validity of a treatment tailored for one group. Thus, the treatment developed and evaluated in this trial will be culturally sensitive and not tailored to only one culture. As we anticipate a sample 
composed predominantly of African American and White participants, this approach should enable the resulting treatment to be disseminated effectively, particularly to these two groups.

\subsection{Inclusion/Exclusion Criteria}

Inclusion-To be eligible for participation in this trial, interested individuals must be female and between the ages of 13 and 18. They must also speak English, and meet criteria for LOC-Eating Disorder [as defined by Tanofsky-Kraff and colleagues [22] or BED in children, as defined by Marcus and Kalarchian [21].

Exclusion-Exclusion criteria include: Alcohol or drug dependence in the last three months, current significant suicidal ideation or self-harm behaviors reported during the assessment periods, developmental disability or neurological impairment that would impair the ability of the participant to benefit from the intervention, or psychosis, including schizophrenia, or bipolar I disorder. Appropriate clinical referrals will be made if adolescents are ineligible because of a potential mental health issue. Also, if it is suspected or discovered that a potential participant is a victim of abuse or neglect, clinicians will respond in accordance with state laws. Adolescents will not be excluded who are receiving other treatments for their eating problem, rather this will be documented and considered in the analyses.

\subsection{Recruitment and Retention}

Participants will be recruited from local pediatricians and pediatric specialists. In addition, we will inform area schools, churches, community based organizations and PTAs about the LIBER8 program and will post flyers in grocery stores and health clubs. These advertisements will encourage interested individuals to contact the study research coordinator. During their initial contact, potential participants will complete a screening questionnaire to determine if they meet study criteria. The screening questionnaire also includes demographic questions.

We will implement several strategies to minimize attrition, including the use of incentives. All participants (i.e., intervention and control groups) will be given $\$ 25$ gift cards for completing assessments (preassessment, post-assessment and the three month follow-up assessment). Adolescents who attend the final session will be given Certificates of Completion, and the final session will include a graduation ceremony symbolizing participants' commitment to a healthy lifestyle. Parents/caregivers will be invited to this portion of the last session. Additional measures used to minimize attrition include asking participants to provide at least two phone numbers where they can be reached (preferably parents'/caregivers' cell and work numbers), as well as an email address. Reminder calls will be made for all appointments. At least six attempts will be made (at several times of day and night) to reschedule missed assessment appointments. The number of sessions attended by intervention participants will also be recorded and considered in the analyses. 


\subsection{Assessment Schedule}

Potential participants will complete several measures during the phone screening process (see Table 1 for list of all measures). This information will be used to determine initial eligibility. If participants meet these initial criteria, families will be invited to complete a series of baseline questionnaires/interviews to confirm eligibility and gather baseline outcome data. Next, participants will be randomly assigned to either the LIBER8 intervention or 2BFit, a weight management control group. Mid-way through treatment, participants in both groups will complete the Eating Disorder Examination Questionnaire [18]. Immediately following the completion of the intervention, and again three months later, both groups will complete the same questionnaires/interviews collected at baseline (as summarized in Table 1). Intervention feasibility and acceptability will also be evaluated on a weekly basis (both by participants and group leaders). Finally, weekly self-harm screenings will be conducted in both groups throughout the active phase of the study. Participants who develop significant suicidal intention, clinically significant self-harming behaviors, bulimia nervosa, or anorexia nervosa during the course of the study will be referred to appropriate treatment and withdrawn from the study.

Use of this experimental design offers protection from several threats to the study's internal validity. In particular, use of a pretest will enable us to control for pre-intervention individual differences in scores on the dependent measures. Additional internal validity threats such as regression to the mean and selection are controlled by the use of random assignment [71]. Contamination of other treatment effects is also controlled for by random assignment and in data analysis where other treatments will be considered as covariates. Moreover, because both the intervention and control groups are assessed at the end of the intervention, factors such as maturation, history, and testing effects are controlled [71]. Differential attrition by group will be minimized by using the same recruitment and retention strategies for each group.

\subsection{Assessment Instruments}

Please refer to Table 1 for the assessment schedule.

\section{Screening Measures}

Loss of Control Eating Disorder Screening Questionnaire (LOC-ED): During the initial phone screen interview, adolescents will be administered the LOC-ED by a trained research assistant. This measure was initially designed to be self-report, but to streamline the screening process and reduce barriers to participation, items were administered in an interview format. The LOC-ED assesses binge and LOC eating frequency and related characteristics [22]. Examples of these related characteristics include hunger and satiety levels, emotions, mood, and compensatory behaviors.

Mini-Neuropsychiatric Interview version 6.0 (MINI): The M.I.N.I. [72] is a brief structured clinical interview designed to assess the presence of Axis I disorders as described in the Diagnostic and Statistical Manual, $4^{\text {th }}$ edition (DSM-IV-TR) [73]. The M.I.N.I. will be used to assess the following symptoms: anorexia nervosa, bulimia nervosa, alcohol and substance abuse, suicide, and self-harm (the latter two of which will be assessed in-person 
during baseline assessments rather than on the phone, for safety reasons). This information will be used to determine whether participants meet eligibility criteria, and to evaluate for severe pathology throughout the duration of the intervention. The M.I.N.I. has evidenced sufficient and similar reliability and validity when compared with other diagnostic screening tools such as the Composite International Diagnostic Interview (CIDI) and the Structured Clinical Interview for DSM-IV [72].

\section{Feasibility and Acceptability}

Measures of Recruitment Feasibility: Feasibility of recruitment for this pilot trial will be assessed via detailed tracking of efforts to recruit (e.g., numbers of pediatricians and other healthcare providers contacted, number of organizations where flyers are posted, number of persons who call the research office, etc). We will also record information regarding the scope of recruiting efforts needed to acquire the proposed sample size (e.g., the number of phone calls and emails made to research staff inquiring about the study, the number of screened individuals who are eligible for the study). We will also record all the reasons for ineligibility among screened individuals. We will make at least six attempts to re-contact people who were eligible but did not attend the baseline assessment session to determine their reasons for not continuing in the study.

Measures of Treatment Acceptability and Retention Feasibility: Feasibility of retaining an adequate sample for this pilot intervention will be assessed via detailed tracking of attendance at each session (for both groups). When a session is missed, we will call and inquire as to the reason and track these reasons. In addition, we will assess participants' satisfaction with the intervention to which they are assigned by having them complete a satisfaction survey at the end of each session. Retention feasibility will also be assessed by re-contacting [on at least six occasions] individuals who drop out of either intervention at any point. We will assess participants' reasons for dropping out, and ask structured, openended questions regarding their suggestions for improving the feasibility and acceptability of the intervention.

Measures of Intervention Feasibility: Feasibility of the interventions will be assessed via protocol fidelity monitoring. Intervention feasibility will also be evaluated via weekly questionnaires completed by the therapists (in both groups). These questionnaires will assess therapists' perceptions of: (1) the feasibility of completing all the material included in the manual for that particular session; (2) the appropriateness of the topics reviewed, and (3) participants' perceived responses to the material.

Feasibility of texting (LIBER8 group only) will be evaluated by tracking: (1) the number of participants who have unlimited texting at the time of enrollment (which will inform budgeting for a larger trial); (2) participants' adherence to the texting protocol and reported reasons for non-compliance (including missing responses or failure to respond to texts initiated by study staff); (3) information regarding lost or stolen cell phones and the number of replacements needed; (4) participants' baseline use of and comfort with texting as a means of communication, and (5) participants' use of and comfort with texting at the end of the intervention. 
Exit Questionnaire: Following completion of the interventions, participants will evaluate the acceptability of their assigned group via an exit questionnaire. This questionnaire consists of items rated on a 1-5 scale and open-ended questions assessing: what participants liked and disliked about the intervention, their thoughts about the duration and number of sessions, as well as the topics covered, perceived benefits of their participation, comfort level, overall satisfaction, and suggestions for improvement.

\section{Intervention Outcomes}

Eating Disorders Examination (EDE): The overeating section of the EDE [23] is a semistructured clinical interview that assesses LOC eating behaviors including: objective binge eating episodes (OBEs, or overeating with LOC), subjective binge eating episodes (SBEs, or perceived overeating with LOC), and objective overeating (overeating without LOC), occurring within the previous month. The EDE has been found to yield more valid results regarding binge eating behaviors than the EDE-Q, a self-report questionnaire, in studies of adolescents $[74,75]$. This measure has also yielded reliable and stable scores in previous studies $[23,76]$.

Self-monitoring: Self-monitoring of LOC and overeating behaviors will begin after the first LIBER8 session and continue for the entire intervention period. Each night, participants will be expected to text the system before bedtime and input their data for the four symptoms (i.e., number of LOC episodes, number of overeating episodes, urges to overeat, and average intensity of urges to overeat). After the message is received, the participant will receive an automated feedback message comparing the present data to that of the previous night. Responses will be based on three categories: status quo, improved, deteriorated (e.g., Good job in overeating less even though your urges remained the same). If at 9 am the following morning, there has been no input, participants will receive a text prompt to input their data. Later in the day, participants who have not responded (or were late doing so) will be prompted via text to provide information regarding their barriers to texting (or to doing so promptly). This information will be explored in sessions as a means of problem-solving barriers to self-monitoring. Participants will also receive short "Tips of the Day" throughout the duration of LIBER8. These messages will complement the topic reviewed in that week's session.

Eating Disorder Examination Questionnaire (EDE-Q): The EDE-Q [18] will be used to assess disordered eating behaviors and cognitions. The EDE-Q is a 36-item self-report questionnaire adapted from the EDE [23]. It includes four subscales (Restraint, Shape Concern, Weight Concern, and Eating Concern) and has evidence of good reliability and validity $[77,78]$. To enhance the validity of this measure, a one-page handout will provide participants with definitions and examples of the different types of eating episodes, as is recommended [79].

Child Depression Inventory (CDI): The CDI [80] will be used to assess symptoms of depression. This version of the CDI was validated with 10-18-year-olds, has been used with African American adolescents, and its reliability and validity are well-established [81, 82]. 
Revised Child Anxiety and Depression Scales (RCADS): Items from the RCADS [83] were formulated to be consistent with diagnostic criteria in the DSM-IV [73]. Anxiety will be evaluated with the five anxiety subscales: separation anxiety disorder, social phobia, obsessive-compulsive disorder, panic disorder, and generalized anxiety disorder. This measure has demonstrated good psychometric properties with community and clinical adolescent samples [83, 84].

Emotional Eating Scale - Adapted for Children (EES-C): The EES-C is a 25-item measure that will assess participants' desire to eat as a means of coping with an array of negative emotions, including anger, sadness, and anxiety [85]. This measure has demonstrated good internal consistency, convergent and discriminant validity, and temporal stability with children and adolescents [85].

Pediatric Quality of Life Inventory (PedsQL): Quality-of-life will be assessed using the PedsQL [86, 87]. This measure is widely used with adolescents and assesses perceptions of how health affects daily life in four areas: physical, emotional, social, and school. The PedsQL yields reliable scores and effectively discriminates between healthy children and those with health problems $[86,88]$.

Cognitive Emotion Regulation Questionnaire (CERQ): Emotion regulation will be assessed using the CERQ [89]. The CERQ was developed to assess cognitions following experiences of negative life events. The CERQ was validated in an adolescent sample [90], and scores on this measure are associated with internalizing psychopathology among adolescents [91].

Barratt Impulsiveness Scale-11 (BIS-11): Impulsivity will be assessed using the BIS-11 [92]. The BIS-11 is a 30-item self-report measure of trait impulsivity. This measure yields internally consistent scores [93]. Scores on this measure have been associated with measures of planning and impulse control [93].

\section{Eating in the Absence of Hunger Questionnaire for Children and Adolescents (EAH-}

C): The frequency of eating when satiated will be assessed using the EAH-C [94]. The EAH-C was validated in a sample of youth ages 6-19 (nearly a third of whom were African American) [94]. The measure yielded internally consistent and temporally stable scores. Further, it manifested convergent validity with measures of emotional and LOC eating.

Height and Weight: Weight will be assessed using a digital scale and a stadiometer will be used to assess height.

Demographic Questionnaire: This questionnaire will collect information regarding participants' date of birth, year in school, their comfort with texting and current texting use. 


\subsection{Interventions}

\subsection{LIBER8 Intervention}

We anticipate that LIBER8 will be a 12 session group intervention designed to be delivered in weekly, 1.5 hour sessions. The exact format and duration of the intervention will be strongly influenced by input from the focus groups. LIBER8 will be grounded in CBT, but will also integrate skills training derived from DBT. Moreover, based on previous research and the results of the focus groups, the intervention will be culturally sensitive; specifically it will encourage participants to reflect on how their own cultural backgrounds and experiences have shaped their attitudes and behaviors regarding emotions and their expression, eating, body image and self-care. Moreover, materials including pictures and songs will reflect the diversity of our participants [e.g., session on music as a coping strategy will use music identified by teens]. The interactive and interdependent nature of the skill building activities are also consistent with relational cultural values of girls and African Americans [95].

The emphasis on skills training is particularly relevant for adolescents, as several authors have suggested that the effectiveness of disease-specific interventions is limited, because they do not adequately address factors that promote resiliency in individuals with disordered eating [96, 97], but rather, primarily focus on modifying specific unhealthy behaviors, such as bingeing. LIBER8 aims not only to reduce eating disorder-specific behaviors, but also to increase overall resilience factors, as is recommended [98].

Although the exact content and format of the sessions will be determined in Stage 1, based on prior research and the investigators' collective professional experiences in this area, we propose the outline presented in Table 2.

Topics will build upon one another; thus, topics introduced earlier in the intervention will be referred back to throughout (e.g., CBT model, self-monitoring). In addition, participatory activities will be incorporated throughout the intervention, including group discussions, role plays, and introduction of a novel coping skill in each session [99, 100]. Many activities will occur within small dyads and will then be brought back to the larger group, as adolescents are generally more comfortable with this approach than with initially sharing in a larger group. It is important to incorporate participatory experiences such as these into the intervention to enhance overall efficacy via guided skills practice in the natural environment $[99,100]$. In addition, homework will be assigned between sessions so that the skills learned can be practiced [100].

\subsection{Weight Management Control Group}

The control group, 2BFit, will be a behaviorally-based weight management intervention. This specific type of control group was selected based on empirical data which show that, although weight management interventions do not specifically address LOC eating, participants in these types of interventions tend to manifest reductions in bulimic symptoms $[101,102]$. The content of this program will be based upon standardized, empirically based treatments for weight management in adolescents [103-105]. For example, participants will set individualized diet and exercise goals. Nutrition recommendations will be based upon the 
guidelines established by the U.S. Department of Agriculture and Health and Human Services (i.e., MyPlate) [106]. Exercise guidelines will be based on the recommendations of the U.S. Department of Health and Human Services [107]. Participants will also be encouraged to reduce their sedentary behaviors and use strategies such as portion control to improve their dietary habits. In addition to reviewing these recommendations and tracking participant adherence to their goals, sessions will address barriers to change and discuss strategies for overcoming these barriers. Information will be presented and reviewed via interactive activities and discussions.

\subsection{Therapist and Training}

Therapists will be $\mathrm{PhD}$ level clinical psychologists or highly experienced master's level clinicians with experience in the provision of manualized psychotherapy for eating disorders. They will familiarize themselves with the treatment manual and read specified preparatory material. An initial two-day workshop will be conducted at the beginning of the study for all therapists involved. This training will consist of didactic instruction and roleplaying. LIBER8 and 2BFit therapists will attend separate clinical training and supervision sessions, and will be directed not to read or discuss each other's manuals or treatment process. Therapists in both groups will be blinded to the study's hypotheses. Care will be taken by the investigators to ensure that there is no overlap between the two interventions' manuals. An independent rater will review both manuals prior to the training.

\subsection{Data Analysis}

Most of our hypotheses involve continuous response variables assessed longitudinally. If this was a larger (non-pilot) study, we would use random coefficient models, a specific type of mixed model. However, with only 25 participants per group in this treatment development grant, it would be unwise to attempt to fit sophisticated models to this small amount of data, estimating many parameters relative to the sample size, and leaving a relatively small number of error degrees of freedom with which to estimate error variability. In general, the goals of our modeling and testing will be to examine whether or not our results are generally in the predicted direction, and to estimate effect sizes and variation (to be able to provide preliminary data for a more definitive study if the pilot trial suggests that the intervention is feasible, acceptable, and appears to be effective).

\section{Missing data}

We will examine the missing at random assumption by assessing differences between dropouts and completers at baseline as well as response variables up to point of premature withdrawal. If the missing at random assumption does not appear to be tenable, we will report our results but spend additional effort characterizing treatment effect at time of premature withdrawal. 


\subsection{Statistical Analysis for the Specific Aims}

\section{Aim 1}

To develop and standardize a modular manualized intervention [LIBER8] for adolescents with binge or LOC eating behavior to facilitate therapist training and treatment dissemination. In Aim 1 we will develop LIBER8 based on the results of focus groups. This will involve a systematic and careful content analysis of the qualitative data provided by these groups. Several strategies will be used to enhance the validity of the data derived from the focus groups. Sessions will be videotaped and transcribed, and content analysis will be used to identify and code the major themes in the data [108].

\section{Aim 2}

To implement LIBER8 in a small randomized trial to determine feasibility and acceptability. To determine the feasibility and acceptability of LIBER8 in Aim 2, we will calculate: 1) the proportion of individuals who participate in screening who are eligible for the study; 2) the proportion of individuals who are eligible but declines participation; 3) the proportion eligible that schedules pre-testing but does not attend the baseline assessment; and 4) the proportion that completes pretesting but drops out of the intervention. We will also track recruitment sources and identify the most fruitful sites.

Feasibility and acceptability of LIBER8 (and the 2BFit condition) will also be assessed via descriptive analyses (i.e., frequencies, means, standard deviations) of the ordinal-level items on both the satisfaction surveys completed weekly by intervention participants, and the exit questionnaire. These analyses will also be conducted for the therapists' ratings of feasibility and acceptability. Both participants' and therapists' responses to the open-ended questions will be compiled for a content analysis. In addition to evaluating participants' satisfaction with the intervention and assessment process and procedures, we will collect frequency data regarding the number of study measures completed at each time point, the amount of missing data (including missed text responses) and the number of sessions attended by participants. We will also evaluate whether attrition rates varied between the LIBER8 and 2BFit groups. We will also compare the data obtained on each measure of feasibility and acceptability to that from available clinical trials conducted with demographically and clinically similar samples on outcomes such as recruitment, attrition, and satisfaction. All of these data will provide important information useful in refining the intervention and associated assessments/procedures prior to their implementation in a larger-scale RCT.

\section{Aim 3}

To evaluate whether LIBER8 is superior to a Weight Management control for improving targeted disordered eating outcomes (of binge and LOC eating and eating disorder cognitions).

\section{Aim 4}

To evaluate whether adolescents in LIBER8 show reductions in psychiatric symptoms (depression, anxiety, impulsivity, and poor QOL) commonly associated with LOC and binge eating. For Aims 3 and 4, $t$-tests will be conducted at baseline to check for any significant 
differences between the LIBER8 and 2BFit groups. The hypotheses of this study will be tested using a series of repeated measures ANCOVAs, controlling for the influence of pretest scores and other potential covariates, such as BMI. ANCOVA is a particularly useful statistical technique for studies using a pretest-posttest design with random assignment, because it enhances statistical power by reducing error variance [109]. In these analyses, time (of assessment) will be the within-subjects variable, and group (intervention vs. control) will be the between-subjects variable. Outcomes will be adolescents' scores at posttesting and 3-month follow-up. Outcome measures will be transformed if they do not meet the assumptions of normality. In addition, to examine the effects of text-message based selfmonitoring and feedback in symptom improvement, we will use graphical displays and descriptive statistics to quantify differences on change between the first two and the last two weeks on measures of binge and LOC eating behaviors (and urges), eating disorder cognitions, and secondary outcomes of depression, anxiety, QOL, and impulsivity. We will examine pre-post differences using paired $t$-tests and McNemar's test for continuous and categorical variables, respectively.

\subsection{Data Management and Safety Monitoring Plan}

Although the proposed study does not technically require a formal Data and Safety Monitoring Board (DSMB), we have asked an independent clinical-scientist to serve as the study's Data and Safety Monitor. considering the high potential for psychiatric problems in this high-risk group, The DSMB will meet regularly, have access to all protocol data, and will have the authority to inquire into protocol process and to suspend or terminate a protocol. They will also ensure that the study is being conducted according to protocol specifications. The DSMB will receive copies of all reportable adverse events.

Data will be double-entered by the project coordinator and a research assistant with extensive experience in database management. Participants will be assigned a code number, which will be used, instead of names, to maintain confidentiality. Only study staff will have access to identifiable information, and they will be required to complete Health Insurance Portability and Accountability Act (HIPAA) training. In addition, all study staff will be required to complete the Collaborative Investigator Training Initiative (CITI) course in human subjects' protection. All data will be collected and maintained in accordance with these legal and ethical standards. The database will be password-protected, regularly backed up, and maintained on a secure server behind a firewall.

\subsection{Discussion}

LOC eating is prevalent, especially among overweight adolescents, and is associated with psychopathology in this age group [4, 24, 25]. Further, the experience of LOC during eating appears to be more relevant to mental health outcomes in youth than is the reported consumption of a large amount of food that characterizes a classic binge episode. Given the significant number of overweight adolescent girls with LOC eating [6,7], the current study has the potential to impact a substantial proportion of youth at risk for chronic mental health issues. Further, it will integrate two treatment approaches previously shown to yield positive results in adult samples [27, 32, 33, 38], and tailor delivery to African American 
adolescents. Finally, text-messaging will be used to enhance adolescents' engagement in the treatment process and improve adherence to self-monitoring.

Despite the important potential translational implications, implementation of this trial poses several challenges. These include adherence to the text-messaging component of the intervention and cell phone management. As noted above, numerous reminders will be used to maximize adherence to the texting schedule. In addition, to address the potential concern regarding phone management, we carefully considered both our anticipated group size and the cost of plans in our area. At this time, "pay-as-you-go" cellular service is the most costeffective manner in which to obtain all the phones needed during our proposed time period. Having more phones on hand is also much less expensive than paying for the optional insurance provided by most cell carriers. We intend to have 20 cell phones with unlimited texting service on hand for this study. This number was selected because we will be conducting only one LIBER8 group at a time (and anticipate that a total of 25 people will participate in this arm of the study). Thus, we will need a maximum of 12-13 phones at any one time; by having extra phones available we will be able to provide replacements in an extremely timely manner for anyone who loses her phone.

Study phones will be set up so that service will be limited to incoming and outgoing text messages only (i.e., no phone calls will be allowed). Because texting service is unlimited, and calls are not allowed, monthly service fees will remain constant. Participants will be informed during their initial orientation (when they receive their study phone) that any lost or missing cell phones will be immediately deactivated. This deactivation procedure should decrease the likelihood that participants will be tempted to keep the phones. We will also allow individuals who already have unlimited texting prior to enrolling in the study to use their own cell phones during the intervention. Individuals who already have unlimited texting at the time of enrollment will receive an alternative incentive of a $\$ 25$ gift card per month during the course of the intervention. Due to the pilot nature of this study, it is unclear at this point what proportion of our sample will have unlimited texting access at enrollment. Thus, we will assess the use of texting and the number of adolescents who have unlimited texting in both the focus groups and in the pilot intervention. This will provide important feasibility data for the larger RCT.

Another challenge is that the ideal length of this intervention is also not known at this time. Based on our previous experiences with clients with eating concerns in our clinic, we have estimated that the intervention will be approximately 12 weeks in duration. Although LOC eating is a clinical concern with biological, psychological and social etiological factors, all of which could be addressed in greater depth in a longer treatment, we are concerned about balancing treatment intensity with participant burden and feasibility. To evaluate the feasibility and acceptability of this treatment dosage for our target group, we will carefully track attrition and responses to the feasibility measures included in this study.

\subsection{Conclusions}

Results from this project will have immediate applicability for adolescents with LOC eating behaviors and our resultant tested intervention will be ready for implementation in a larger 
RCT. Conducting the pilot work proposed here would provide extremely valuable information regarding the feasibility, acceptability and preliminary efficacy of this approach. In addition, with LIBER8 as our baseline intervention, we will be well-positioned to add and test additional modules focusing on younger children. Finally, with our content fully developed, we will be poised to adapt the intervention to other modes of delivery [e.g., internet plus chat] to enhance disseminability.

\section{Acknowledgments}

Supported by a grant from the National Institutes of Health (R34 MH-086922 (Mazzeo, PI)

\section{References}

1. Hudson JI, Hiripi E, Pope HG Jr. Kessler RC. The prevalence and correlates of eating disorders in the National Comorbidity Survey Replication. Biol Psychiatry. Feb 1.2007 61:348-58. [PubMed: 16815322]

2. Striegel-Moore RH, Cachelin FM, Dohm FA, Pike KM, Wilfley DE, Fairburn CG. Comparison of binge eating disorder and bulimia nervosa in a community sample. Int J Eat Disord. Mar.2001 29:157-65. [PubMed: 11429978]

3. Abbott DW, de Zwaan M, Mussell MP, Raymond NC, Seim HC, Crow SJ, et al. Onset of binge eating and dieting in overweight women: Implications for etiology, associated features and treatment. J Psychosom Res. 1998; 44:367-74. [PubMed: 9587880]

4. Glasofer DR, Tanofsky-Kraff M, Eddy KT, Yanovski SZ, Theim KR, Mirch MC, et al. Binge eating in overweight treatment-seeking adolescents. J Pediatr Psychol. 2007; 32:95-105. [PubMed: 16801323]

5. Isnard P, Michel G, Frelut ML, Vila G, Falissard B, Naja W, et al. Binge eating and psychopathology in severely obese adolescents. Int J Eat Disord. 2003; 34:235-43. [PubMed: 12898560]

6. Decaluwe V, Braet C. Prevalence of binge-eating disorder in obese children and adolescents seeking weight-loss treatment. Int J Obes Relat Metab Disord. 2003; 27:404-9. [PubMed: 12629570]

7. Berkowitz R, Stunkard A, Stallings VA. Binge eating disorder in obese adolescent girls. Annals of the New York Academy of Sciences. 1993; 699:200-6. [PubMed: 8267310]

8. Ross HE, Ivis F. Binge eating and substance use among male and female adolescents. Int J Eat Disord. 1999; 26:245-60. [PubMed: 10441240]

9. Decaluwe V, Braet C, Fairburn CG. Binge eating in obese children and adolescents. Int J Eat Disord. 2003; 33:78-84. [PubMed: 12474202]

10. Johnson WG, Grieve FG, Adams CD, Sandy J. Measuring binge eating in adolescents: Adolescent and parent versions of the questionnaire of eating and weight patterns. Int J Eat Disord. 1999; 26:301-14. [PubMed: 10441246]

11. Ledoux S, Choquet M, Manfredi R. Associated features for self-reported binge eating among male and female adolescents. J Adolesc. 1993; 16:75-91. [PubMed: 8496470]

12. Eddy KT, Tanofsky-Kraff M, Thompson-Brenner H, Herzog DB, Brown TA, Ludwig DS. Eating disorder pathology amoung overweight treatment-seeking youth: Clinical correlates and crosssectional risk modeling. Behav Res Ther. 2007; 45:2360-71. [PubMed: 17509523]

13. Tanofsky-Kraff M, Cohen ML, Yanovski SZ, Cox C, Theim KR, Keil M, et al. A prospective study of psychological predictors of body fat gain among children at high risk for adult obesity. Pediatrics. 2006; 117:1203-1209. [PubMed: 16585316]

14. Tanofsky-Kraff M, Yanovski SZ, Schvey NA, Olsen CH, Gustafson J, Yanovski JA. A prospective study of loss of control eating for body weight gain in children at high risk for adult obesity. Int J Eat Disord. 2009; 42:26-30. [PubMed: 18720473]

15. Field AE, Austin SB, Taylor CB, Malspeis S, Rosner B, Rockett HR, et al. Relation between dieting and weight change amoung preadolescents and adolescents. Pediatrics. 2003; 112:900-6. [PubMed: 14523184] 
16. Tanofsky-Kraff M, Shomaker LB, Olsen C, Roza CA, Wolkoff LE, Columbo KM, et al. A prospective study of pediatric loss of control eating and psychological outcomes. J Abnorm Psychol. 2011; 120:108-18. [PubMed: 21114355]

17. Gormally J, Black S, Daston S, Rardin D. The assessment of binge eating severity among obese persons. Addictive Behaviors. 1982; 7:47-55. [PubMed: 7080884]

18. Fairburn CG, Beglin SJ. Assessment of eating disorders: Interview or self-report questionnaire. Int J Eat Disord. 1994; 16:363-70. [PubMed: 7866415]

19. Bryant-Waugh RJ, Cooper PJ, Taylor CJ, Lask BD. The use of the Eating Disorder Examination with children: A pilot study. Int J Eat Disord. 1996; 19:391-7. [PubMed: 8859397]

20. Goldschmidt AB, Aspen VP, Sinton MM, Tanofsky-Kraff M, Wilfley DE. Disordered eating attitudes and behaviors in overweight youth. Obesity [Silver Spring]. 2008; 16:257-64.

21. Marcus MD, Kalarchian MA. Binge eating in children and adolescents. Int J Eat Disord. 2003; 34:S47-S57. [PubMed: 12900986]

22. Tanofsky-Kraff M, Marcus M, Yanovski SZ, Yanovski JA. Loss of control eating disorder in children age 12 years and younger: proposed research criteria. Eat Behav. 2008; 9:360-5. [PubMed: 18549996]

23. Fairburn, CG.; Cooper, Z. The Eating Disorder Examination. In: Fairburn, CG.; Wilson, TG., editors. Binge eating: Nature, assessment, and treatment. 12th ed. Guilford; New York: 1993. p. 317-60.

24. Goossens L, Braet C, Decaluwe V. Loss of control over eating in obese youngsters. Behav Res Ther. Jan.2007 45:1-9. [PubMed: 16516139]

25. Tanofsky-Kraff M, Faden D, Yanovski SZ, Wilfley DE, Yanovski JA. The perceived onset of dieting and loss of control eating behaviors in overweight children. Int J Eat Disord. 2005; 38:112-22. [PubMed: 16134103]

26. Goldschmidt AB, Jones M, Manwaring JL, Luce KH, Osborne MI, Cunning D, et al. The clinical significance of loss of control over eating in overweight adolescents. Int J Eat Disord. 2008; 41:153-8. [PubMed: 18095271]

27. Linehan, MM. Cognitive-behavioral treatment of borderline personality disorder. Guildford Press; New York, NY: 1993.

28. Fairburn, CG.; Marcus, M.; Wilson, GT. Cognitive-behavioral therapy for binge eating and bulimia nervosa: A comprehensive treatment manual. In: Fairburn, CG.; Wilson, GT., editors. Binge eating: nature, assessment, \& treatment. Guilford Press; New York, NY: 1993. p. 361-404.

29. Fairburn CG, Cooper Z, Shafran R. Cognitive behaviour therapy for eating disorders: a "transdiagnostic" theory and treatment. Behav Res Ther. May; 2003 41(5):509-28. [PubMed: 12711261]

30. Westen D. Manualizing manual development. Clinical Psychology: Science and Practice. 2002; 9:416-8.

31. Penn, D.; Judge, AM.; Jamieson, P.; Garczynski, J.; Hennessey, M.; Romer, D. Treatments for Adolescents that Work. Seligman, M., editor. Oxford Press; London: In Press

32. Wilson GT. Psychological treatment of eating disorders. Ann Rev Clinical Psyc. 2005; 1:439-65.

33. Hay PJ, Bacaltchuk J, Stefano S. Psychotherapy for bulimia nervosa and binging. Cochrane Database Syst Rev. 2004 CD000562.

34. Brownley KA, Berkman ND, Sedway JA, Lohr KN, Bulik CM. Binge eating disorder treatment: A systematic review of randomized controlled trials. Int J Eat Disord. 2007; 40:337-48. [PubMed: 17370289]

35. March JS, Silva S, Petrycki S, Curry J, Wells K, Fairbank J, et al. The treatment for adolescent depression study [TADS]: Long-term effectiveness and safety outcomes. Arch Gen Psychiatry. 2007; 64:1132-43. [PubMed: 17909125]

36. Telch CF, Agras WS. Obesity, binge eating, and psychopathology: Are they related. Int J Eat Disord. 1994; 15:53-61. [PubMed: 8124327]

37. Heatherton TF, Baumeister RF. Binge eating as escape from self-awareness. Psychol Bull. 1991; 110:86-108. [PubMed: 1891520] 
38. Wiser S, Telch CF. Dialectical behavior therapy for binge eating disorder. J Clinical Psyc. 1999; 55:755-68.

39. Lock JD. Adjusting cognitive behavior therapy for adolescents with bulimia nervosa: Results of case series. Am J Psychother. 2005; 59:267-81. [PubMed: 16370133]

40. Smith BA, Schuchman M. Problems of nonadherence in chronically ill adolescents: Strategies for assessment and intervention. Current Opinions in Pediatrics. 2005; 17:613-8.

41. Smith BA, Wood BL. Psychological factors affecting disease activity in children and adolescents with cystic fibrosis: Medical adherence as a mediator. Curr Opin Pediatr. 2007; 19:553-8. [PubMed: 17885474]

42. Jones M, Luce KH, Osborne MI, Taylor K, Cunning D, Doyle AC, et al. Randomized, controlled trial of an internet-facilitated intervention for reducing binge eating and overweight in adolescents. Pediatrics. 2008; 121:453-62. [PubMed: 18310192]

43. Franklin VL, Waller A, Pagliari C, Greene SA. A randomized controlled trial of Sweet Talk, a textmessaging system to support young people with diabetes. Diabetic Med. 2006; 23:1332-8. [PubMed: 17116184]

44. Ferrerr-Roca O, Cardenas A, Diaz-Cardama A, Pulido P. Mobile test phone messaging in the management of diabetes. J Telemed Telecare. 2004; 10:282-5. [PubMed: 15494086]

45. Kwon HS, Cho JH, Kim HS, Lee JH, Song BR, Oh JA, et al. Development of web-based diabetic patient management system using short message service [SMS]. Diabetes Res Clin Pr. 2004; 66:S133-S7.

46. Rodgers A, Corbett T, Bramley D, Riddell T, Willis M, Lin RB, et al. Do you smoke after txt? Results of a randomized trial of smoking cessation using mobile phone text messaging. Tob Control. 2005; 14:255-61. [PubMed: 16046689]

47. Bielli E, Carminati F, La Capra S, Lina M, Brunelli C, Tamburini M. A Wireless Health Outcomes Monitoring System [WHOMS]: Development and field testing with cancer patients using mobile phones. BMC Medical Informatics and Decision Making. 2004; 4:7. [PubMed: 15196308]

48. Madell DE, Muncer SJ. Control over social interactions: An important reason for young people's use of the internet and mobile phones for communication. CyberPsychology \& Behavior. 2007; 10:137-40. [PubMed: 17305461]

49. Walston J, Lissitz R. Computer-mediated focus groups. Evaluation Review. 2000; 24:457-83. [PubMed: 11183483]

50. Kramish CM, Meier A, Carr C, Enga Z, James AS, Reedy J. Health behavior changes after colon cancer: a comparison of findings from face-to-face and on-line focus groups. Fam and Community Health. 2001; 24:88-103.

51. Wadden, TA. The treatment of obesity. In: Stunkard, AJ.; Wadden, TA., editors. Obesity: Theory and Therapy. Raven Press; New York: 1993. p. 197-217.

52. Yanovski SZ, Devlin MJ. Role of behavioral therapy in the management of obesity. Endocrine Practice. 1995; 1:340-5. [PubMed: 15251581]

53. O'Neil PM. Assessing dietary intake in the management of obesity. Obes Res. 2001; 9:361S-6S. [PubMed: 11918342]

54. Anderson B, Ho J, Brackett J, Finkelstein D, Laffel L. Parental involvement in diabetes management rasks: Relationships to blood glucose monitoring, adherence, and metabolic control in young adolescents with insulin-dependent diabetes mellitus. J Pediatr. 1997; 130:257-65. [PubMed: 9042129]

55. Covey N. Breaking teen myths. Consumer Insight. 2009:22-5.

56. Ryan P, Lauver DR. The efficacy of tailored interventions. Journal of Nursing Scholarship. 2002; 34:331-7. [PubMed: 12501736]

57. Bull FC, Holt CL, Kreuter MW, Clark EM, Scharff D. Understanding the effects of printed health education materials: Which features lead to which outcomes? J Health Commun. 2001; 6:265-79. [PubMed: 11550593]

58. Striegel-Moore RH, Dohm FA, Kraemer HC, Taylor CB, Daniels S, Crawford PB, et al. Eating disorders in White and Black women. Am J Psychiatry. 2003; 160:1326-31. [PubMed: 12832249]

59. Hoek HW. Incidence, prevalence and mortality of anorexia nervosa and other eating disorders. Current Opinion in Psychiatry. 2006; 19:389-98. [PubMed: 16721169] 
60. Striegel-Moore RH, Wilfley DE, Pike KM, Dohm FA, Fairburn CG. Recurrent binge eating in Black American women. Archives of Family Medicine. 2000; 9:83-7. [PubMed: 10664648]

61. Taylor JY, Caldwell CH, Baser RE, Faison N, Jackson JS. Prevalence of eating disorders among Blacks in the National Survey of American Life. Int J Eat Disord. 2007; 40:10-4.

62. French SA, Story M, Neumark-Sztainer D, Downes B, Resnick M, Blum R. Ethnic differences in psychosocial and health behavior correlates of dieting, purging, and binge eating in a populationbased sample of adolescent females. Int J Eat Disord. 1997; 22:315-22. [PubMed: 9285269]

63. American Psychological Association Task Force on Resilience and Strength in Black Children and Adolescents. Resilience in African American children and adolescents: A vision for optimal development. American Psychological Association; 2008. Available from: http:// www.apa.org/pi/cyf/resilience.html [cited 2009 November 1]

64. Belgrave FZ, Reed MC, Plybon LE, Corneille M. The impact of a culturally enhanced drug prevention program on drug and alcohol refusal efficacy among urban African American girls. J Drug Educ. 2004; 34:267-79. [PubMed: 15648887]

65. Jackson-Gilfort A, Liddle HA, Tejeda MJ, Dakof GA. Facilitating engagement of African American male adolescents in family therapy: A cultural theme process study. J Black Psychol. 2001; 27:321-40.

66. Airhihenbuwa CO, Kumanyika S, Agurs TD, Lowe A, Saunders D, Morssink CB. Cultural aspects of African American eating patterns. Ethn Health. 1996; 1:245-60. [PubMed: 9395569]

67. James DC. Factors influencing food choices, dietary intake, and nutrition-related attitudes among African Americans: application of a culturally sensitive model. Ethn Health. 2004; 9(4):349-67. [PubMed: 15570680]

68. Blixen CE, Singh A, Thacker H. Values and beliefs about obesity and weight reduction among African American and Caucasian women. J Transcult Nurs. 2006; 17:290-7. [PubMed: 16757669]

69. Blixen CE, Singh A, Xu M, Thacker H, Mascha E. What women want: Understanding obesity and preferences for primary care weight reduction interventions among African-American and Caucasian women. J Natl Med Assoc. 2006; 98:1160-70. [PubMed: 16895288]

70. Ammerman A, Leung MM, Cavallo D. Addressing disparities in the obesity epidemic. North Carolina Medical Journal. 2006; 67:301-4. [PubMed: 17066662]

71. Cook, TD.; Campbell, DT. Quasi-experimentation: Design and analysis issues for field settings. Houghton Mifflin; Boston: 1979.

72. Sheehan DV, Lecrubier Y, Sheehan KH, Amorim P, Janavs J, Weiller E, et al. The MiniInternational Neuropsychiatric Interview [M.I.N.I.]: the development and validation of a structured diagnostic psychiatric interview for DSM-IV and ICD-10. J Clin Psychiatry. 1998; 59:22-33. [PubMed: 9881538]

73. American Psychiatric Association. Diagnostic and statistical manual of mental disorders. 4th ed., text-revision. American Psychiatric Association; Washington, DC: 2000.

74. Black C, Wilson TG. Assessment of eating disorders: Interview versus questionnaire. Int J Eat Disord. 1996; 20:43-50. [PubMed: 8807351]

75. Binford RB, Le Grange D, Jellar CC. Eating Disorders Examination versus Eating Disorders Examination-Questionnaire in adolescents with full and partial-syndrome bulimia nervosa and anorexia nervosa. Int J Eat Disord. 2005; 37:44-9. [PubMed: 15690465]

76. Williamson DA, Lawson OJ, Brooks ER, Wozniak PJ, Ryan DH, Bray GA, et al. Association of body mass with dietary restraint and disinhibition. Appetite. 1995; 25:31-41. [PubMed: 7495325]

77. Luce KH, Crowther JH. The reliability of the Eating Disorder Examination-self-report questionnaire version [EDE-Q]. Int J Eat Disord. 1999; 25:349-51. [PubMed: 10192002]

78. Mond JM, Hay PJ, Rodgers B, Owen C, Beumont PJ. Temporal stability of the Eating Disorder Examination Questionnaire. Int J Eat Disord. Sep.2004 36:195-203. [PubMed: 15282689]

79. Goldfein JA, Devlin MJ, Kamenetz C. Eating Disorder Examination-Questionnaire with and without instruction to assess binge eating in patients with binge eating disorder. Int $\mathrm{J}$ Eat Disord. 2005; 37:107-11. [PubMed: 15732081]

80. Kovacs M. The Children's Depression Inventory. Psychopharmacological Bulletin. 1985; 21:9958. 
81. Zeller MH, Modi AC. Predictors of health-related quality of life in obese youth. Obesity. 2006; 14:122-30. [PubMed: 16493130]

82. Hayward C, Gotlib IH, Schraedley PK, Litt IF. Ethnic differences in the association between pubertal status and symptoms of depression in adolescent girls. J Adolesc Health. 1999; 25:143-9. [PubMed: 10447041]

83. Chorpita BF, Yim L, Moffitt CE, Umemoto LA, Francis SE. Assessment of symptoms of DSM-IV anxiety and depression in children: A revised child anxiety and depression scale. Behav Res Ther. 2000; 38:835-55. [PubMed: 10937431]

84. Chorpita BF, Moffitt CE, Gray J. Psychometric properties of the revised child anxiety and depression scale in a clinical sample. Behav Res Ther. 2005; 43:309-22. [PubMed: 15680928]

85. Tanofsky-Kraff M, Theim KR, Yanovski SZ, Bassett AM, Burns NP, Ranzenhofer LM, et al. Validation of the emotional eating scale adapted for use in children and adolescents [EES-C]. Int $\mathrm{J}$ Eat Disord. Apr.2007 40:232-40. [PubMed: 17262813]

86. Varni JW, Seid M, Kurtin PS. PedsQL 4.0: Reliability and validity of the Pediatric Quality of Life Inventory Version 4.0 generic core scales in healthy and patient populations. Medical Care. 2001; 39:800-12. [PubMed: 11468499]

87. Varni JW, Seid M, Rode CA. The PedsQL-super [TM]: Measurement model for the Pediatric Quality of Life Inventory. Medical Care. 1999; 37:126-39. [PubMed: 10024117]

88. Varni JW, Burwinkle TM, Katz ER, Meeske K, Dickinson P. The PedsQL in pediatric cancer: Reliability and validity of the Pediatric Quality of Life Inventory Generic Core Scales, Multidimensional Fatigue Scale, and Cancer Module. Cancer. 2002; 94:2090-106. [PubMed: 11932914]

89. Garnefski, N.; Kraaij, V.; Spinhoven, P. Manual for the use of the Cognitive Emotion Regulation Questionnaire [CERQ]. DATEC; Leiderdorp, the Netherlands: 2002.

90. Garnefski N, Kraaij V, Spinhoven P. Negative life events, cognitive emotion regulation and emotional problems. Pers Indiv Diff. 2001; 30:1311-27.

91. Garnefski N, Kraaij V, van Etten M. Specificity of relations between adolescents' cognitive emotion regulation strategies and Internalizing and externalizing psychopathology. J Adolesc. 2005; 28:619-31. [PubMed: 16203199]

92. Patton JH, Stanford MS, Barratt ES. Factor structure of the Barratt impulsiveness scale. J Clinic Psychol. 1995; 51:768-74.

93. Pietrzaka RH, Spraguea A, Snyder PJ. Trait impulsiveness and executive function in healthy young adults. J Res Pers. 2008; 42:1347. 1351.

94. Tanofsky-Kraff M, Ranzenhofer LM, Yanovski SZ, Schvey NA, Faith M, Gustafson J, et al. Psychometric properties of a new questionnaire to assess eating in the absence of hunger in children and adolescents. Appetite. 2008; 51:148-55. [PubMed: 18342988]

95. Belgrave, FZ. African American girls: Reframing perceptions and changing experiences. Springer; New York, NY: 2009.

96. Mussell MP, Binford RB, Fulkerson JA. Eating disorders: Summary of risk factors, prevention programming, and prevention research. Counsel Psychol. 2000; 28:764-96.

97. Striegel-Moore R, Schreiber GB, Lo A, Crawford P, Obarzanek E, Rodin J. Eating disorder symptoms in a cohort of 11 to 16-year-old African American and white girls: The NHLBI growth and health study. Int J Eat Disord. 1998; 27:49-66. [PubMed: 10590449]

98. Levine, MP.; Piran, N.; Stoddard, C. Mission more probable: Media literacy, activism, and advocacy as primary prevention. In: Piran, N.; Levine, MP.; Steiner-Adair, C., editors. Preventing eating disorders: A handbook of interventions and special challenges. Brunner/Mazel; Philadelphia: 1999. p. 3-25.

99. Neumark-Sztainer D, Sherwood NE, Coller T, Hannan PJ. Primary prevention of disordered eating among preadolescent girls: Feasibility and short-term effect of a community-based intervention. J Am Diet Assoc. 2000; 100:1466-73. [PubMed: 11138438]

100. Stewart DA, Carter JC, Drinkwater J, Hainsworth J, Fairburn CG. Modification of eating attitudes and behavior in adolescent girls: A controlled study. Int J Eat Disord. 2001; 29:107-18.

[PubMed: 11429973] 
101. Stice E, Rohde P, Seeley J, Gau J. Brief cognitive-behavioral depression prevention program for high-risk adolescents outperforms two alternative interventions: A randomized efficacy trial. J Consult Clin Psychol. 2008; 76:595-606. [PubMed: 18665688]

102. Stice E, Trost A, Chase A. Healthy weight control and dissonance-based eating disorder prevention programs: Results from a controlled trial. Int J Eat Disord. 2003; 33:10-21. [PubMed: 12474195]

103. Shrewsbury VA, O'Connor J, Steinbeck KS, Stevenson K, Lee A, Hill AJ, et al. A randomised controlled trial of a community-based healthy lifestyle program for overweight and obese adolescents: the Loozit® study protocol. BMC Public Health. 2009; 9:119. [PubMed: 19402905]

104. Epstein LH, Myers MD, Raynor HAS, B. E. Treatment of pediatric obesity. Pediatrics. 1998; 101:554-70. [PubMed: 12224662]

105. Janicke DM. Treating Pediatric Obesity. Arch Pediatr Adolesc Med. 2008; 162:1191-2. [PubMed: 19047549]

106. U.S. Department of Agriculture. U.S. Department of Health and Human Services. Dietary Guidelines for Americans. U.S. Government Printing Office; Washington, DC: Dec. 2010

107. Department of Health and Human Services [HHS]. The Dietary Guidelines for Americans. U. S. Department of Agriculture [USDA]; 2005.

108. Knodel, J. The design and analysis of focus group studies: A practical approach. In: Morgan, DL., editor. Successful focus groups: Advancing the state of the art. Sage; Newbury Park, CA: 1993. p. 35-50.

109. Huck SW, McLean RA. Using a repeated measures ANOVA to analyze the data from a pretestposttest design: A potentially confusing task. Psychol Bull. 1975; 82:511-8. 
Table 1

Assessment Schedule

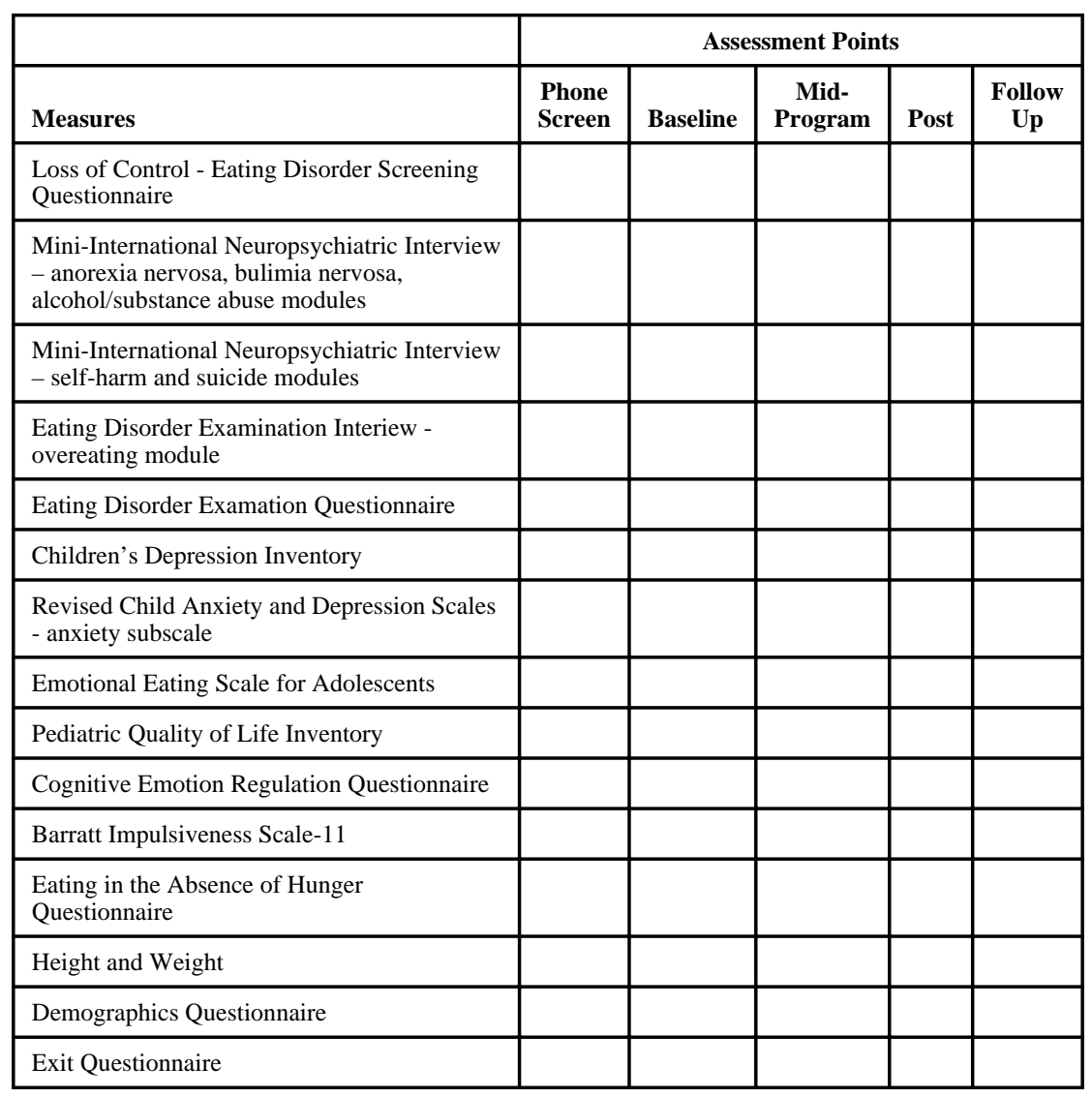




\section{Table 2}

\section{Proposed LIBER8Session Outline}

\begin{tabular}{|l|l|}
\hline $\begin{array}{l}\text { Session } \\
\text { uumber }\end{array}$ & Session Topic \\
\hline Session 1 & Introduction: Defining LOC eating and introducing text-messaging \\
\hline Session 2 & Mindful Eating and Nutrition \\
\hline Session 3 & Identifying Emotions \\
\hline Session 4 & Emotion Regulation \\
\hline Session 5 & Thoughts and Feelings (Introduction to CBT Model) \\
\hline Session 6 & Thoughts and Feelings II (CBT model and Cognitive Distortions) \\
\hline Session 7 & Advanced Cognitive Coping skills (e.g. self-soothing) \\
\hline Session 8 & Social Skills and Assertiveness Training I \\
\hline Session 9 & Food Pressures and Assertiveness Training II \\
\hline Session 10 & Body Image and Media Literacy \\
\hline Session 11 & Flex session \\
\hline Session 12 & $\begin{array}{l}\text { Summary and relapse prevention with parent education and completion } \\
\text { celebration }\end{array}$ \\
\hline
\end{tabular}

